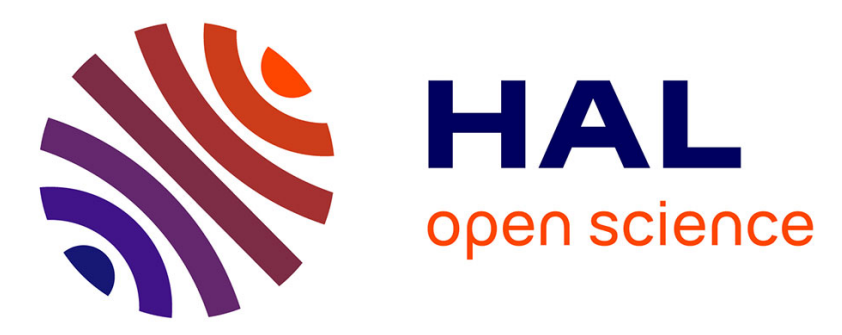

\title{
Suivi en continu de la qualité d'un cours d'eau: apports et difficultés des méthodes optiques
}

Philippe Namour

\section{To cite this version:}

Philippe Namour. Suivi en continu de la qualité d'un cours d'eau : apports et difficultés des méthodes optiques. 7ème Colloque Interdisciplinaire en Instrumentation, Jan 2016, Saint-Nazaire, France. hal01280225

\section{HAL Id: hal-01280225 \\ https://hal.science/hal-01280225}

Submitted on 29 Feb 2016

HAL is a multi-disciplinary open access archive for the deposit and dissemination of scientific research documents, whether they are published or not. The documents may come from teaching and research institutions in France or abroad, or from public or private research centers.
L'archive ouverte pluridisciplinaire HAL, est destinée au dépôt et à la diffusion de documents scientifiques de niveau recherche, publiés ou non, émanant des établissements d'enseignement et de recherche français ou étrangers, des laboratoires publics ou privés. 


\title{
Suivi en continu de la qualité d'un cours d'eau : apports et difficultés des méthodes optiques
}

\author{
P. Namour ${ }^{1,4}$, M.N. Pons 2 , S. Wachs ${ }^{3}$, X. France 3 \\ ${ }^{1 / n s t i t u t ~ d e s ~ S c i e n c e s ~ A n a l y t i q u e s, ~ U M R ~ C N R S ~ 5280, ~} 5$ rue de la Doua, 69100 \\ Villeurbanne \\ 2Laboratoire Réactions et Génie des Procédés, UMR CNRS 7274, Université de Lorraine, \\ BP 20451, 54001 Nancy cedex \\ ${ }^{3}$ GEMCEA, 149 rue Gabriel Péri, 54500, Vandoeuvre-les-Nancy \\ 4 Irstea, 5, rue de la Doua, 69100, Villeurbanne \\ marie-noelle.pons@univ-lorraine.fr
}

\begin{abstract}
In-situ monitoring of river surface water: contributions and difficulties of optical methods
\end{abstract}

Optical sensors, namely a submersible UV-visible spectrophotometer, a fluorescence probe for dissolved organic matter and a fluorimeter with a circulation cuvette dedicated to tryptophan-like fluorescence measurement have been deployed for the real-time monitoring of a periurban stream. Biofouling was a main issue for the UV-visible spectrophotometer and the tryptophan-like fluorimeter. However nitrates variation could be monitored by UV-vis spectroscopy based on the maximum of the second derivative of the spectrum in the UVrange.

\section{Introduction}

Depuis plusieurs années, les techniques optiques (néphélométrie, spectrométrie UV-visible, fluorescence) sont considérés comme prometteuses dans l'élaboration d'outils de caractérisation de la qualité des eaux (eaux de surface (douces, marines, estuariennes) ou souterraines) [1, 2]. Leur mise en œuvre a l'avantage de ne pas nécessiter l'emploi de réactifs dont la gestion (stockage, déchets liquides) n'est pas aisée surtout dans le cas d'un déploiement dans une zone isolée. Cependant il est souvent reproché aux capteurs optiques des problèmes d'encrassement liés au développement d'un biofilm sur les surfaces optiques.

Dans le cadre d'un large projet de surveillance et de restauration d'un cours d'eau péri-urbain fortement anthropisé et dégradé, différents capteurs optiques basés sur la spectrométrie UV-visible et la fluorescence ont été mis en œuvre dans une station de mesure pendant plusieurs mois. Les mesures en ligne ont été comparées à des analyses en laboratoire ainsi qu'à des informations fournies par des analyseurs (nitrates et azote ammoniacal) et capteurs ( $\mathrm{pH}$, conductivité) également en ligne. 


\section{Matériels et méthodes}

Le Grémillon est un cours d'eau périurbain fortement dégradé, affluent en rive droite de la Meurthe sur le territoire du Grand Nancy. Depuis plusieurs années l'évolution de sa qualité en fonction de l'avancée des travaux de restauration est suivie à l'aide d'une station de mesure située dans la partie avale du bassin versant. Le cours d'eau y est enterré. Un débitmètre à ultrasons aérien (DU100, Hendress-Hauser) permet d'en connaître le débit, à partir d'une loi de conversion hauteur d'eau / débit. L'eau est d'abord pompée dans un bac dans lequel se trouvent les sondes de $\mathrm{pH}$ et de conductivité (Figure 1). Dans ce bac ont également été immergés un spectrophotomètre UV-visible submersible (S::can, Aix-en-Provence) avec un chemin optique de $1 \mathrm{~cm}$ et une sonde permettant de mesurer la fluorescence de la matière organique dissoute (MOD) $\left(\lambda_{\mathrm{ex}}=370 / \lambda_{\mathrm{em}}=460 \mathrm{~nm}\right)$ (modèle $\mathrm{ECO}$, Wetlabs, Philomath, Oregon). La fenêtre optique de la sonde Wetlabs est protégée par un volet en cuivre qui n'est ouvert que lors de la mesure. Le spectrophotomètre enregistre un spectre dans la gamme 220- $732.5 \mathrm{~nm}$ (pas de $2.5 \mathrm{~nm}$ ) stocké dans un module con::stat d'où les données sont récupérées régulièrement.

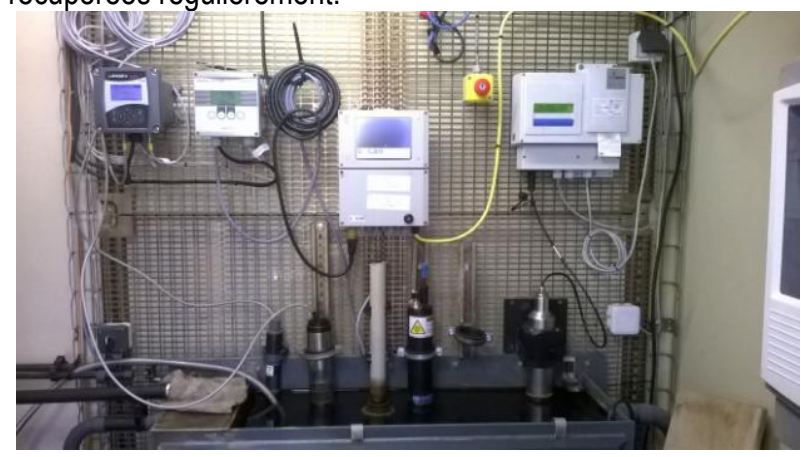

Figure 1 : Intérieur de la station de mesure avec le bac intermédiaire dans lequel sont plongés les différents capteurs.

Des pompes permettent d'alimenter deux analyseurs en ligne pour le dosage de l'azote ammoniacal et des nitrates. Une étape de filtration est nécessaire pour l'analyseur à azote ammoniacal et le liquide filtré est également utilisé par un fluorimètre SMF4 (Safe Training Systems Ltd, Wokingham, Grande-Bretagne). Cet appareil, doté d'une LED émettant dans I'UV, permet de suivre la fluorescence de type tryptophane. II est équipé d'une petite cellule en quartz alimentée par des tubulures de diamètre interne $2 \mathrm{~mm}$. Le fluorimètre SMF4 a été étalonné avec des solutions de tryptophanes dans la gamme $0-100 \mu \mathrm{g} / \mathrm{kg}$. Pour tous ces appareils, la fréquence d'acquisition est de $15 \mathrm{~min}$, sauf pour la sonde Wetlabs pour laquelle plusieurs périodes d'échantillonnage ont été testées. La station est maintenue régulièrement (deux visites par semaine) avec nettoyage de toutes les sondes. Les tests ont été conduits du 1 avril au 31 août 2015.

Un préleveur automatique permet d'obtenir des échantillons analysables au laboratoire (en particulier en termes de carbone organique dissous, azote total dissous et azote 
ammoniacal). Le carbone organique dissous et l'azote total dissous sont dosés à l'aide d'un VCHS (Shimadzu, Noisiel). L'azote ammoniacal est dosé par la méthode de Nessler. Des spectres UV-visible et de fluorescence synchrone sont également collectés sur un spectrophotomètre Anthélie Light (Secoman) et un fluorimètre FL-2500 (Hitachi) avec des cuvettes en quartz (longueur de chemin optique $=1 \mathrm{~cm}$ ). Les spectres de fluorescence synchrone sont réalisés avec un décalage de $50 \mathrm{~nm}$ entre les longueurs d'onde d'excitation et d'émission.

\section{Résultats}

\subsection{Spectrophotomètre submersible}

La Figure 2 présente les variations du débit du cours d'eau, de la conductivité et de l'absorbance à $255 \mathrm{~nm}$. Celle-ci est extraite du spectre fourni par le spectrophotomètre submersible S::can. Du fait de l'imperméabilisation du bassin versant et des pentes notamment en rive droite, le ruisseau réagit très rapidement et fortement en cas de précipitations. Chaque précipitation induit une baisse de la conductivité. Pour certaines d'entre elles une augmentation rapide de $A_{25}$ est observée. L'augmentation progressive de l'absorbance est attribuable à la formation d'un biofilm sur les surfaces optiques, en dépit du nettoyage bihebdomadaire.

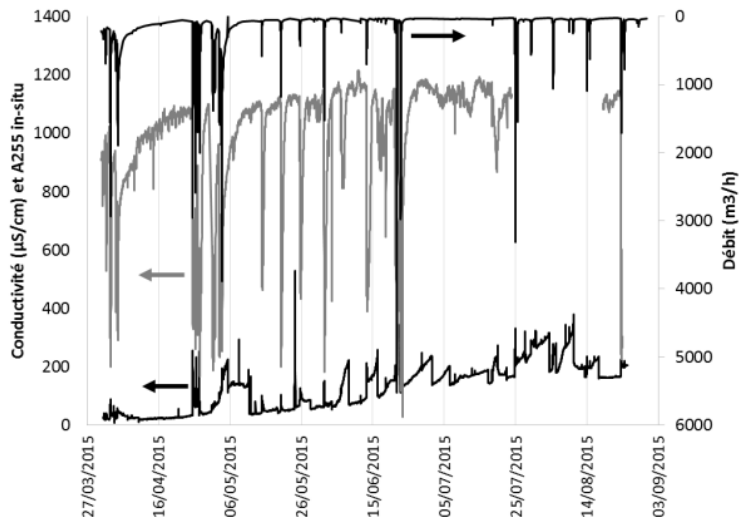

Figure 2 : Débit du cours d'eau, conductivité et $A_{255}$ (spectrophotomètre submersible)

La méthode développée par Ferree et Shannon [3] pour estimer la teneur en nitrates à partir du maximum de la dérivée seconde du spectre UV-visible entre 200 et $250 \mathrm{~nm}$ a été appliquée. Dans le cas présent ce maximum est observé à la longueur d'onde de $227.5 \mathrm{~nm}$. On observe une très bonne corrélation en dépit de la dérive du spectrophotomètre. Par exemple, un coefficient de détermination de 0.95 a été obtenu pour le mois d'avril 2015. 
a)

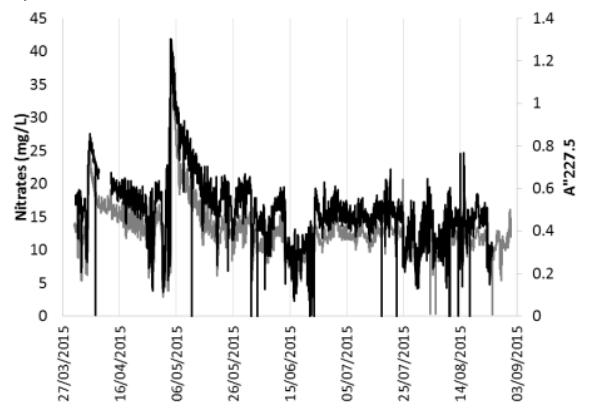

b)

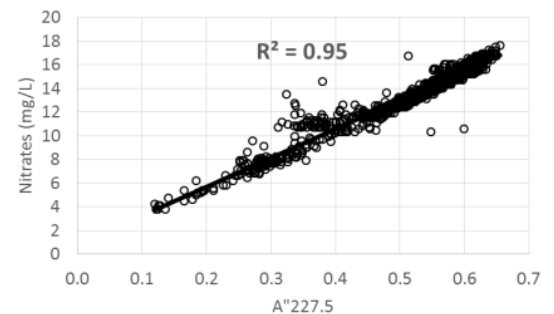

Figure 3 : Comparaison des évolutions de la concentration en nitrates mesurée à la station de mesure et de la dérivée seconde à $227.5 \mathrm{~nm}$ (A"227.5) extraite des spectres du spectrophotomètre submersible S::can (a). Corrélation entre A"227.5 et la concentration en nitrates pendant le mois d'avril 2015 (b).

\subsection{Suivi de la matière organique dissoute}

Plusieurs périodes d'échantillonnage ont été testées sur la sonde Wetlabs ( 1 min, 5 min et $15 \mathrm{~min}$ ) afin de voir quel était le meilleur compromis entre le suivi d'un évènement de crue et l'autonomie de la sonde (Figure 4). Une période d'échantillonnage de 1 min a été ainsi choisie pour le mois de juin 2015. La concentration de la matière organique dissoute varie brutalement à chaque évènement pluvieux. La Figure 5 a rassemble le signal brut de la sonde Wetlabs, l'absorbance à $255 \mathrm{~nm}$ issue du spectrophotomètre submersible S::can et le débit du cours d'eau sur une quinzaine de jours. On voit nettement que la sonde Wetlabs n'est pas affectée par une dérive due au développement d'un biofilm sur la surface optique. La superposition sur deux jours consécutifs de temps sec (Figure $5 b$ ) montre la bonne reproductibilité du signal, qui semble corrélé à celui de l'azote ammoniacal. Des entrées d'eau usée non traitée sont suspectées sur le cours d'eau dans sa partie amont, ce qui conduit à des valeurs d'azote ammoniacal non conformes à la règlementation dans le cours d'eau.

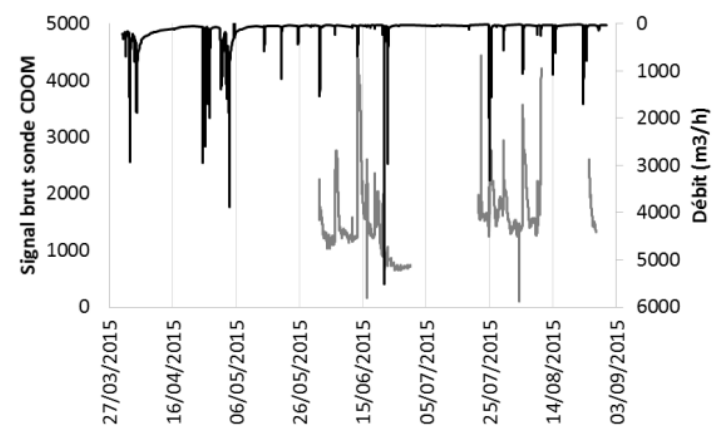

Figure 4 : Suivi global de la matière organique dissoute et du débit du Grémillon 
a)

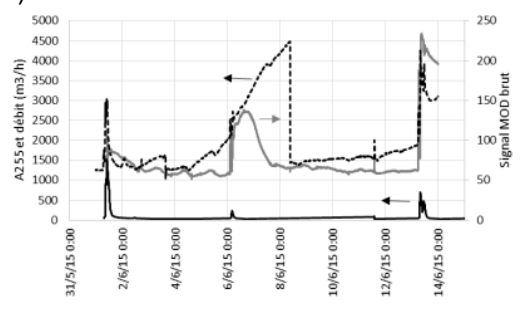

b)

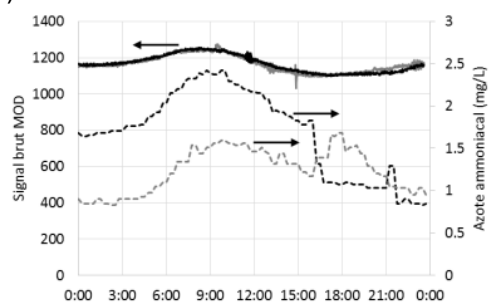

Figure 5: Evolution du signal brut de la matière organique dissoute (MOD), de l'absorbance à $255 \mathrm{~nm}\left(\mathrm{~A}_{255}\right)$ et du débit du Grémillon (a), du signal brut de la matière organique dissoute et de l'azote ammoniacal (b).

\subsection{Comparaison avec des échantillons ponctuels}

La Figure 6 compare les évolutions de l'absorbance à $255 \mathrm{~nm}$ issue du spectrophotomètre submersible $\mathrm{S}$ :.can $\left(\mathrm{A}_{255}\right)$ et la MOD provenant de la sonde Wetlabs avec les mesures effectuées sur les prélèvements sur 24h par temps sec (débit du Grémillon $=50$ $\mathrm{m}^{3} / \mathrm{h}$ ) : absorbance à $255 \mathrm{~nm}$ ( $\mathrm{A}_{255}$ labo), carbone organique dissous (COD) et intensité de fluorescence synchrone pour une longueur d'onde d'excitation de $350 \mathrm{~nm}\left(\mathrm{~F}_{350}\right)$. Cette longueur d'onde permet de suivre l'évolution de la fluorescence de la matière organique dissoute de type humique. II n'y a pas de variation très remarquable des différentes grandeurs. On observe cependant que $A_{255}$ labo, MOD et COD décroissent légèrement au cours de ces $24 \mathrm{~h}$, alors que $\mathrm{A}_{255}$ augmente légèrement, du fait du dépôt sur les fenêtres optiques.

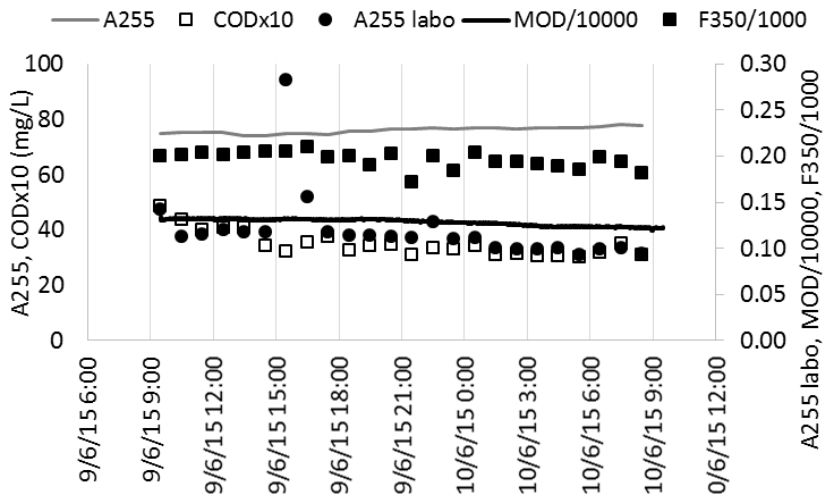

Figure 6 : Evolution de l'absorbance à $255 \mathrm{~nm}$ issue du spectrophotomètre submersible ( $\left.\mathrm{A}_{255}\right)$ et du signal brut de la sonde Wetlabs (MOD), du carbone organique dissous (COD), de l'absorbance à $255 \mathrm{~nm}$ mesurée au laboratoire ( $\mathrm{A}_{255}$ labo) et de l'intensité de fluorescence synchrone $\left(\mathrm{F}_{350}\right)$ pendant $24 \mathrm{~h}$ par temps sec. 


\subsection{Fluorescence de type tryptophane}

Le fonctionnement du fluorimètre SMF4 avait été préalablement validé en laboratoire, sur des échantillons discrets [4]. Sa mise en œuvre en continu s'est avérée délicate du fait du développement rapide de biofilm sur les parois optiques, même si l'appareil avait été placé préventivement derrière un système de filtration. Ce phénomène est d'autant plus important du fait du faible diamètre des tubulures et de la difficulté à nettoyer la cellule. Des enregistrements ont cependant pu être effectués en avril, quand le phénomène n'était pas encore très développé. La Figure 7 montre les variations de la concentration en tryptophane pendant quatre jours. L'appareil de mesure de l'azote ammoniacal étant en maintenance pendant cette période, une comparaison des deux signaux n'est pas possible. Une périodicité de $24 \mathrm{~h}$ est détectable sur la concentration en tryptophane, avec un maximum vers $6 \mathrm{~h}$ du matin.

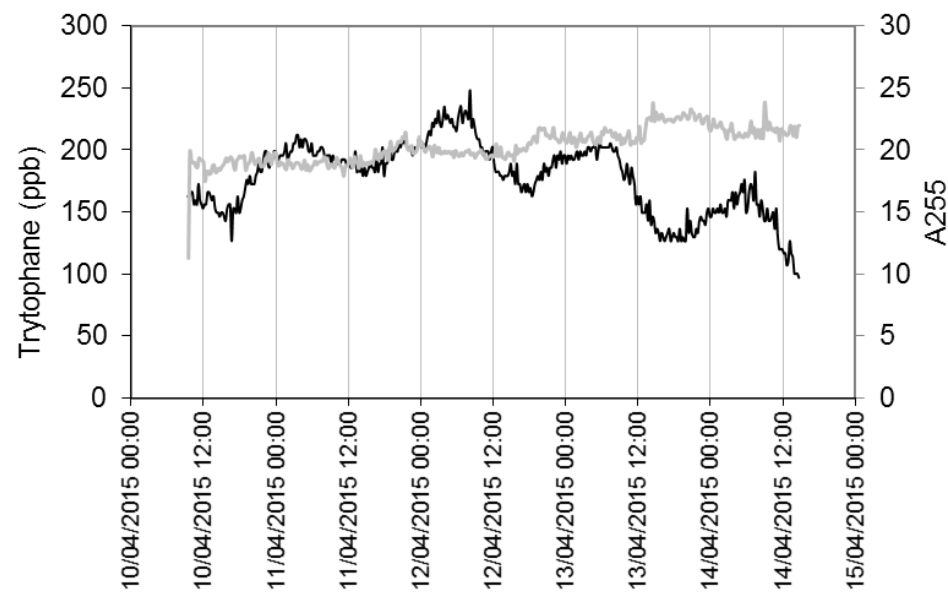

Figure 7 : Variations de la concentration en tryptophane et de l'absorbance à $255 \mathrm{~nm}$ issue du spectrophotomètre submersible $A_{255}$

\section{Conclusions}

Le but de ce travail était d'évaluer les difficultés de mise en œuvre de plusieurs capteurs optiques pour le suivi de la qualité de l'eau de surface d'un ruisseau impacté par une pollution anthropique. Les principaux problèmes rencontrés sont liés à l'encrassement des parois optiques :

- Cet encrassement a causé une dérive du spectrophotomètre UV-visible submersible S::can, en dépit d'un nettoyage bihebdomadaire à la brosse douce. Cependant cette perte de sensibilité n'a pas affectée le suivi de la concentration en nitrates à partir de la dérivée seconde.

- Aucun encrassement n'a été observé pour la sonde fluorimétrique Wetlabs pour le suivie de la matière organique dissoute : cela est certainement dû au fait que la surface optique n'est exposée au milieu liquide que lors de la mesure et que le 
volet de protection est en cuivre. II faut cependant ajuster sa période d'échantillonnage à son autonomie (ou la relier à une alimentation en continu)

- Le fluorimètre SMF4 ne peut pas dans sa configuration actuelle être utilisé en continu car l'encrassement est observé trop rapidement et la cellule de mesure est très difficile à nettoyer.

\section{Remerciements}

Les auteurs remercient l'ANR pour son aide financière dans le cadre du projet EPEC.

\section{Bibliographie}

[1] Pons MN, Potier O, Pontvianne S, Laurent N, France X, Battaglia P, Spectrophotometric characterization of human impacted surface waters in the Moselle watershed. Water Science \& Technology. 2011;64(3):602-9.

[2] Assaad A, Pontvianne S, Corriou JP, Pons MN, Spectrophotometric characterization of dissolved organic matter in a rural watershed: the Madon River (N-E France). Environmental Monitoring and Assessment. 2015;187(4):88.

[3] Gutierrez A, Zhang Y, Assaad A, France X, Adouani N, Pons MN, Assessment of field fluorometers. Water Science \& Technology, 2014;70(8): 1335-40.

[4] Ferree MA, Shannon RD, Evaluation of a second derivative UV/visible spectroscopy technique for nitrate and total nitrogen analysis of wastewater samples. Water Research. 2001;35(1):32732. 